\title{
Ischemic stroke induces cardiac dysfunction and alters transcriptome profile in mice
}

Jie Chen ${ }^{1,2,3 \dagger}$, Jiahong Gong ${ }^{1,2+}$, Haili Chen ${ }^{1,2+}$, Xuqing Li Li, $^{1,}$, Li Wang ${ }^{1,2}$, Xiaoli Qian ${ }^{1,2}$, Kecheng Zhou ${ }^{1,2}$, Ting Wang ${ }^{1,2}$, Songhe Jiang ${ }^{1,2+}$, Lei $\mathrm{Li}^{4 \dagger}$ and Shengcun $\mathrm{Li}^{1,2,4^{*}+}$

\begin{abstract}
Background: Stroke can induce cardiac dysfunction in the absence of primary cardiac disease; however, the mechanisms underlying the interaction between the neurological deficits and the heart are poorly understood. The objective of this study was to investigate the effects of stroke on cardiac function and to identify the transcriptome characteristics of the heart.

Results: Stroke significantly decreased heart weight/tibia length ratio and cardiomyocyte cross-sectional areas and increased atrogin-1 and the E3 ubiquitin ligase MURF-1, indicating myocardial atrophy in MCAO-induced mouse hearts. RNA sequencing of mRNA revealed 383 differentially expressed genes (DEGs) in MCAO myocardium, of which 221 were downregulated and 162 upregulated. Grouping of DEGs based on biological function and quantitative PCR validation indicated that suppressed immune response and collagen synthesis and altered activity of oxidoreductase, peptidase, and endopeptidase may be involved in MCAO-induced cardiomyopathy. The DEGs were mainly distributed in the membrane or extracellular region of cardiomyocytes and acted as potential mediators of stroke-induced cardiac dysregulation involved in cardiac atrophy.
\end{abstract}

Conclusion: Stroke induced a unique transcriptome response in the myocardium and resulted in immediate cardiac atrophy and dysfunction.

Keywords: Ischemic stroke, Cardiac dysfunction, Atrophy, Transcriptome

\section{New and noteworthy}

Our study revealed three new major findings: (1) experimental induced stroke caused cardiac dysfunction, (2) atrophic phenotype of the heart occurred after induced stroke, and (3) a unique myocardial mRNA expression profile was produced after induced stroke in mice.

\footnotetext{
* Correspondence: lishengcun@wmu.edu.cn

${ }^{\dagger}$ Jie Chen, Jiahong Gong and Haili Chen contributed equally to this work.

'Shengcun Li, Lei Li and Songhe Jiang are co-senior authors.

${ }^{1}$ Rehabilitation Medicine Center, The Second Affiliated Hospital and Yuying

Children's Hospital of Wenzhou Medical University, Wenzhou 325027,

Zhejiang, China

${ }^{2}$ Integrative \& Optimized Medicine Research center, China-USA Institute for

Acupuncture and Rehabilitation, Wenzhou Medical University, Wenzhou

325027, Zhejiang, China

Full list of author information is available at the end of the article
}

\section{Introduction}

Cardiovascular and cerebrovascular diseases remain among the primary causes of death and medical costs worldwide [1], and share several risk factors [2]. Although it is well known that cardiogenic strokes contribute to the occurrence of ischemic stroke [3], increasing evidence also suggest that elevated cardiac troponin [4] and left atrial enlargement [5] occur in patients with stroke. These cardiac biomarkers (cardiac troponin and left atrial diameter) are associated with the presence of large vessel occlusions [6]. Myocardial injury, ischemialike electrocardiographic changes and arrhythmias, frequently occur in acute stroke patients, even in the absence of primary heart disease [2]. In this study, we define heart disease of central nervous system origin as stroke-induced cardiomyopathy. Cardiac dysfunction

C The Author(s). 2021 Open Access This article is licensed under a Creative Commons Attribution 4.0 International License, which permits use, sharing, adaptation, distribution and reproduction in any medium or format, as long as you give appropriate credit to the original author(s) and the source, provide a link to the Creative Commons licence, and indicate if changes were made. The images or other third party material in this article are included in the article's Creative Commons licence, unless indicated otherwise in a credit line to the material. If material is not included in the article's Creative Commons licence and your intended use is not permitted by statutory regulation or exceeds the permitted use, you will need to obtain permission directly from the copyright holder. To view a copy of this licence, visit http://creativecommons.org/licenses/by/4.0/ The Creative Commons Public Domain Dedication waiver (http://creativecommons.org/publicdomain/zero/1.0/) applies to the data made available in this article, unless otherwise stated in a credit line to the data. 
induced by stroke is discussed in several animal experiments $[7,8]$. Focal cerebral ischemia induces long-term cardiac dysfunction, with reduced left ventricular ejection fraction (EF) and increased left ventricular volumes; these are associated with higher peripheral sympathetic activity [9]. In addition to impairing cardiac contractility, stroke also leads to greater myocardial vulnerability [10]. One study reported that cerebral ischemic stroke in adult mice induces chronic cardiac dysfunction, fibrosis, hypertrophy and secondary immune response, and may contribute to post stroke cardiac dysfunction [11]. Conversely, another study indicated that transient cardiac atrophy, and not fibrosis and hypertrophy, is induced, owing to up-regulation of the E3-ligase atrogin-1 and up-regulation of Pparg-dependent genes [12]. The mechanisms of conventional cardiac injury, such as sympathetic regulation [9], inflammation [11], and autophagy [13] are considered to be involved in the process of cardiomyopathy after stroke. Historically, stroke therapeutic strategies have focused on treating two parts separately; targeting the central nervous system for brain injury, and treating systemic complications as their own entity [14]. Although the previous study has found some differential molecular expression in the heart muscle 14 days after stroke [12], the underlying molecular mechanisms of stroke-induced neurogenic cardiac dysfunction are yet to be established and we did research at a different point in time than the previous study.

Herein, we first analyzed cardiac function and myocardial remodeling in an induced stroke mouse model. We then used next generation RNA sequencing (RNA-seq) technology to investigate the transcriptome change in mouse heart after stroke. Finally, we described and analyzed the bioinformatics functions of differentially expressed genes (DEGs) in the heart muscle. Understanding the direct mechanisms of stroke-induced cardiomyopathy adequately will be valuable for the development of targeted therapies and promoting stroke rehabilitation.

\section{Materials and methods}

\section{Animals and cerebral ischemia model}

All experiments were approved by the Institutional Animal Care and Use Committee of Wenzhou Medical University and performed in accordance with the National Institute of Health's Guide for the Care and Use of Laboratory Animals. Male (8-9 weeks) C57BL/6 mice were purchased from the Model Animal Research Centre of Wenzhou Medical University. Mice were housed at a constant temperature of $22^{\circ} \mathrm{C}$ in a $12 / 12$-light/dark cycle with free access to regular rodent chow and tap water. Mice were randomly assigned to two groups according to the random number table ( $n=6$, each group) as follows: Sham group and MCAO group.
Focal cerebral ischemia was induced by transient middle cerebral artery occlusion (MCAO) for $60 \mathrm{~min}$, as described previously [12, 15]. Briefly, mice were anesthetized with $1.5 \%$ isoflurane in $\mathrm{O}_{2}$. A thermostatic heating blanket was used to maintain core body temperature close to $37^{\circ} \mathrm{C}$ during surgery. After a midline neck incision, a standardized silicone rubber-coated No.6.0 nylon monofilament was inserted into the right carotid artery and advanced via the internal carotid artery to occlude the origin of the middle cerebral artery. After $60 \mathrm{~min}$, mice were re-anesthetized, and the occluding filament was removed to allow reperfusion. Mice subjected to MCAO with neurological deficit scores of 2-3 were used in subsequent experiments. Exclusion criteria of MCAO group are as follows: mice with subarachnoid hemorrhage; TTC staining showed no obvious infarct size in brain tissue section; mice died within $24 \mathrm{~h}$ after operation. Mice with the conditions above need to be excluded. Owing to the mortality rate of about $30 \%$ in MCAO group, the shortfall needs to be made up. We performed these experiments under the ARRIVE (Animal Research: Reporting of In Vivo Experiments) guidelines. Ethical approval for all experimental procedures was granted by the Animal Ethical and Welfare Committee at Wenzhou Medical University.

\section{Cardiac function measurement}

Mice were anesthetized with isoflurane (1\%) and an animal ultrasound system Vevo2100 (VisualSonics, Canada) equipped with a $40 \mathrm{MHz}$ pediatric transducer, which was used to record an echocardiogram. M-mode and 2-D parasternal short-axis scans at the level of the papillary muscles were used to assess changes in the left ventricle (LV) end-systolic inner diameter, LV end-diastolic inner diameter, and fractional shortening (FS, \%) [16, 17]. To assess diastolic function, we obtained apical four-chamber views of the LV. The pulsed wave Doppler measurements of maximal early (E) and late (A) transmitral velocities in diastole were obtained in the apical view with a cursor at the mitral valve inflow [18].

\section{Assessment of neurological deficit and cerebral infarction} Neurological deficit was assessed in each animal at 2 $\mathrm{h}, 24 \mathrm{~h}$ and 4 days following MCAO in a blinded manner using a 4-point evaluation as described previously [19]. Mice subjected to MCAO with neurological deficit scores of 2-3 were used in subsequent experiments. After obtaining the heart, quickly remove the brain of mice. The cerebral infarction was identified using 2, 3, 5-triphenyl-tetrazolium (TTC) staining for sections of brains. . 


\section{Histological analyses}

Cardiac sections sized $5 \mu \mathrm{m}$ were cut from paraffinembedded tissue. H\&E staining was used for pathological evaluation of the hearts. The myocardial collagen was assessed by staining with Picrosirius red (Solarbio, China), the area of total collagen deposition was measured by Image-Pro Plus 6.0 software. Cross-sectional areas of cardiomyocytes were measured by ImageJ software after staining for cell membranes and nuclei with fluorescein isothiocyanate-conjugated wheat germ agglutinin (WGA) (Thermo Fisher Scientific, USA) and Hoechst33342 (Thermo Fisher Scientific, USA), respectively.

\section{RNA-seq data analysis and library construction}

Total RNA was extracted using mirVana miRNA Isolation Kit (Shanghai OE-Biotech) following the manufacturer's protocol [20]. RNA integrity was evaluated using Agilent 2100 Bioanalyzer (Agilent Technologies, Santa Clara, CA, USA). The samples with RNA Integrity Number (RIN) $\geq 7$ were subjected to further analysis. The libraries were constructed using TruSeq Stranded mRNA LT Sample Prep Kit (Illumina, San Diego, CA, USA) according to the manufacturer's protocol. Then, these libraries were sequenced on the Illumina sequencing platform (HiSeqTM 2500 or Illumina HiSeq X Ten) and $125 \mathrm{bp} / 150 \mathrm{bp}$ paired-end reads were generated.

\section{Gene ontology (GO) and network analysis}

Cufflinks quantitatively evaluated the RPKM (reads per kilobase million) value of each gene [21]. HTSeq-count calculated the read counts of each gene [22]. Differential expression analysis was performed using the DESeq $R$ package [23]. The negative binomial distribution test method (NB) was used to verify the significance of the difference in the number of reads. The $P$-value $<0.05$ adjusted by false discovery rate (FDR) and fold change (FC) $>2$ or $<0.5$ were set as the thresholds for significant differential expression. GO enrichment and KEGG (Kyoto Encyclopedia of Genes and Genomes) pathway enrichment analysis [24] of DEGs were respectively performed using $\mathrm{R}$ package, based on the hypergeometric distribution [25]. The number of counts of each sample gene was normalized using DESeq software [6] (basemean value was used to estimate the expression quality), the fold change of difference was calculated, and the number of reads was tested for the significance of difference using NB (negative binomial distribution test). Finally, the differential protein-coding genes were screened based on the fold change of difference and the significance of difference. All protein-coding genes were used as the background list, and the list of differential protein-coding genes was used as the candidate list screened from the background list. The hypergeometric distribution was used to calculate the $P$-value representing whether the GO function was significantly enriched in the list of differential protein-coding genes, and then the $P$-value was corrected by Benjamini \& Hochberg multiple test to obtain the FDR. Pathway analysis of differential protein-coding genes was performed using the KEGG database [7], and the significance of DEG enrichment in each pathway entry was calculated using a hypergeometric distribution.

\section{Quantitative PCR (qPCR)}

Total RNA was isolated from the mouse heart tissue using TRIzol reagent (TaKaRa, Dalian, China), and 1000 ng of RNA was reverse-transcribed to cDNA using the first-strand synthesis system for PCR (Invitrogen, Carlsbad, CA, USA) according to the manufacturer's protocol. The cDNA was used as a template in qPCR to analyze the mRNA expression levels of atrial natriuretic peptide (ANP), beta-myosin heavy chain $(\beta-\mathrm{MHC})$, collagen-1 and -3 , IL-1 $\beta$, IL- 6 and TNF- $\alpha$ genes, along with selected DEGs. The result comparison between sham group and MCAO group was tested by Student's t-test.

All primer sequences used in this study are listed along with their accession numbers in Supplemental Table S1.

\section{Western blotting and ELISA}

Protein levels were analyzed using Western blotting or ELISA according to the instructions.

\section{Statistical analysis}

All data are presented as mean \pm SD. The level of statistical significance was determined using one-way analysis of variance (ANOVA). Student's t-test was used for comparison between two groups. Statistical analyses were performed using GraphPad Prism v5.0 (GraphPad Software, San Diego, CA, USA). A $P$-value $<0.05$ was considered to be statistically significant.

\section{Results}

\section{MCAO-induced cardiac dysfunction}

To gain insight into the potential molecular changes in MCAO-induced cardiac dysfunction model, we first established an in vivo mice model of middle cerebral artery occlusion, and performed cardiac ultrasound on the mice on the first and fourth days, as shown in Fig. 1A. Figure $1 \mathrm{~B}$ and $\mathrm{C}$ show cerebral infarction and neurological deficits following induced cerebral ischemia, indicating that the MCAO model was established successfully in this study. Understanding the pathophysiological changes in the early stages of stroke is important for the development of rehabilitation and treatment strategies. Therefore, we focused on the acute changes in cardiac function after stroke within 1-4 days. 


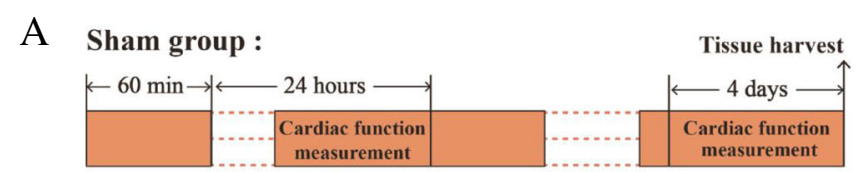

\begin{tabular}{|c|c|c|}
\hline MCAO g & & Tissue harvest \\
\hline$\leftarrow 60 \mathrm{~min}-$ & 24 hours $\longrightarrow$ & -4 days \\
\hline MCAO & \begin{tabular}{|c|}
$\begin{array}{c}\text { Cardiac function } \\
\text { measurement }\end{array}$ \\
\end{tabular} & $\begin{array}{c}\text { Cardiac function } \\
\text { measurement }\end{array}$ \\
\hline
\end{tabular}

B

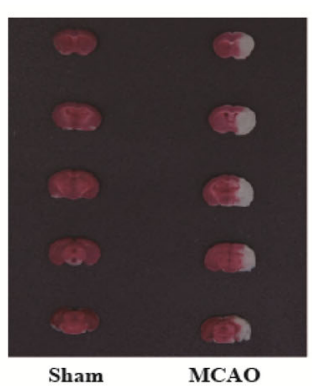

D

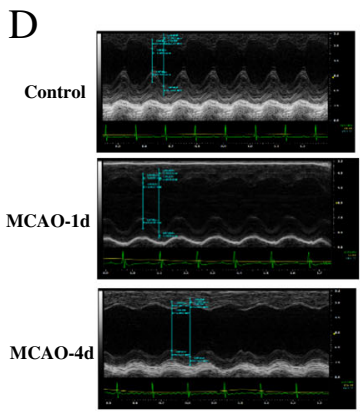

F

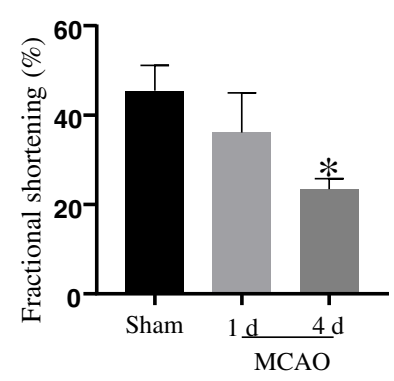

$\mathrm{H}$

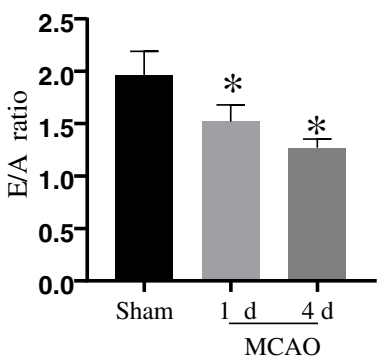

C

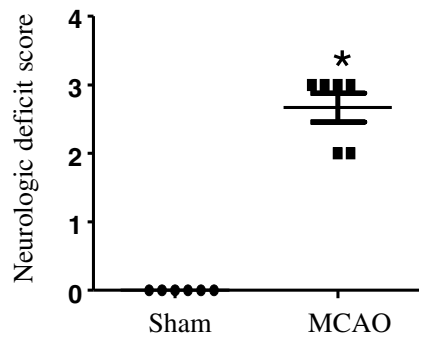

E

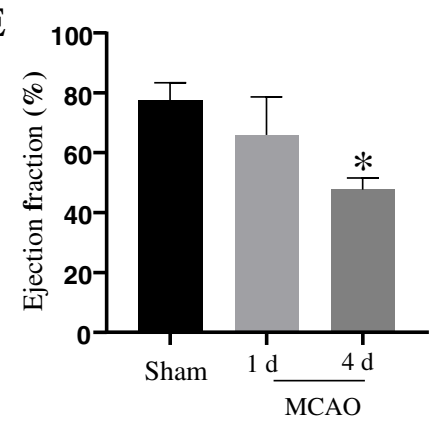

G

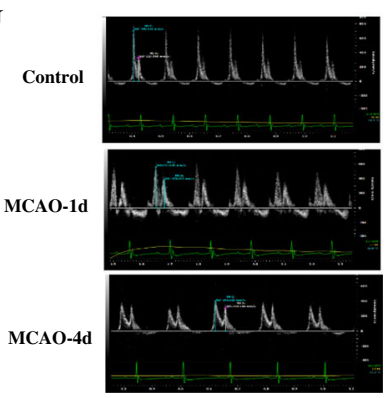

I $\left.{ }^{250}\right] \quad *$

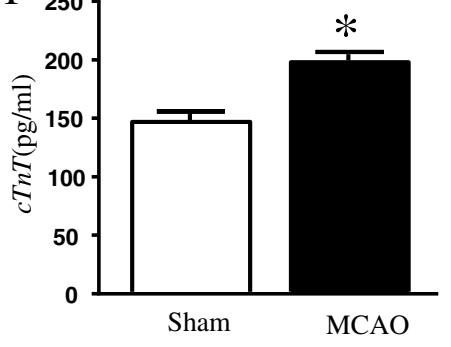

Fig. 1 (See legend on next page.) 
(See figure on previous page.)

Fig. 1 Consequences of stroke on cardiac function. (A) Schematic diagram of the mice models, including sham group and MCAO group; (B) Right hemispheric brain samples were stained with triphenyl tetrazolium chloride (TTC) $24 \mathrm{~h}$ after a 60 min middle cerebral artery occlusion (MCAO). (C) Neurological deficits of MCAO-induced mouse. (D) Representative view at the papillary muscle level of echocardiography (M-model). (E) Ejection fraction (EF, \%) and (F) fractional shortening (FS, \%). (G) A representative mitral E/A image on Doppler echocardiography. (H) E/A ratio. (I) $\mathrm{CTnT}$ level in serum. Data are presented as mean $\pm \mathrm{SD}, n=6 .{ }^{*} P<0.05$ vs sham-operated

Cardiac function impairment was detected in the mice $24 \mathrm{~h}$ after stroke, which further decreased at 4 days compared with that of the sham-operated mice (Fig. 1D). As shown in Fig. $1 \mathrm{E}$ and F, stroke decreased the EF\% and FS\% as markers of systolic function deterioration. Similarly, progressive impairment of diastolic function was identified by a reduced E/A ratio in MCAO mouse hearts (Fig. 1G and $\mathrm{H}$ ). In the initial state of anesthesia, the average heart rate of MCAO group decreased compared to sham group (Supplemental Table S2). cTnT is an important marker of myocardial injury. We used
ELISA to detect the value of cTnT in each group. The results showed that $\mathrm{cTnT}$ was increased in MCAO mouse heart (Fig. 1I).

MCAO led to cardiac atrophy and myocardial remodeling As dramatic weight loss occurred in mice after stroke, we speculated that myocardial atrophy may occur. Heart weight/tibia length ratio was reduced after stroke, compared with the sham-operated mice (Fig. 2A). Histological analysis of cardiomyocyte cross-sectional areas also revealed that cardiomyocyte size decreased in

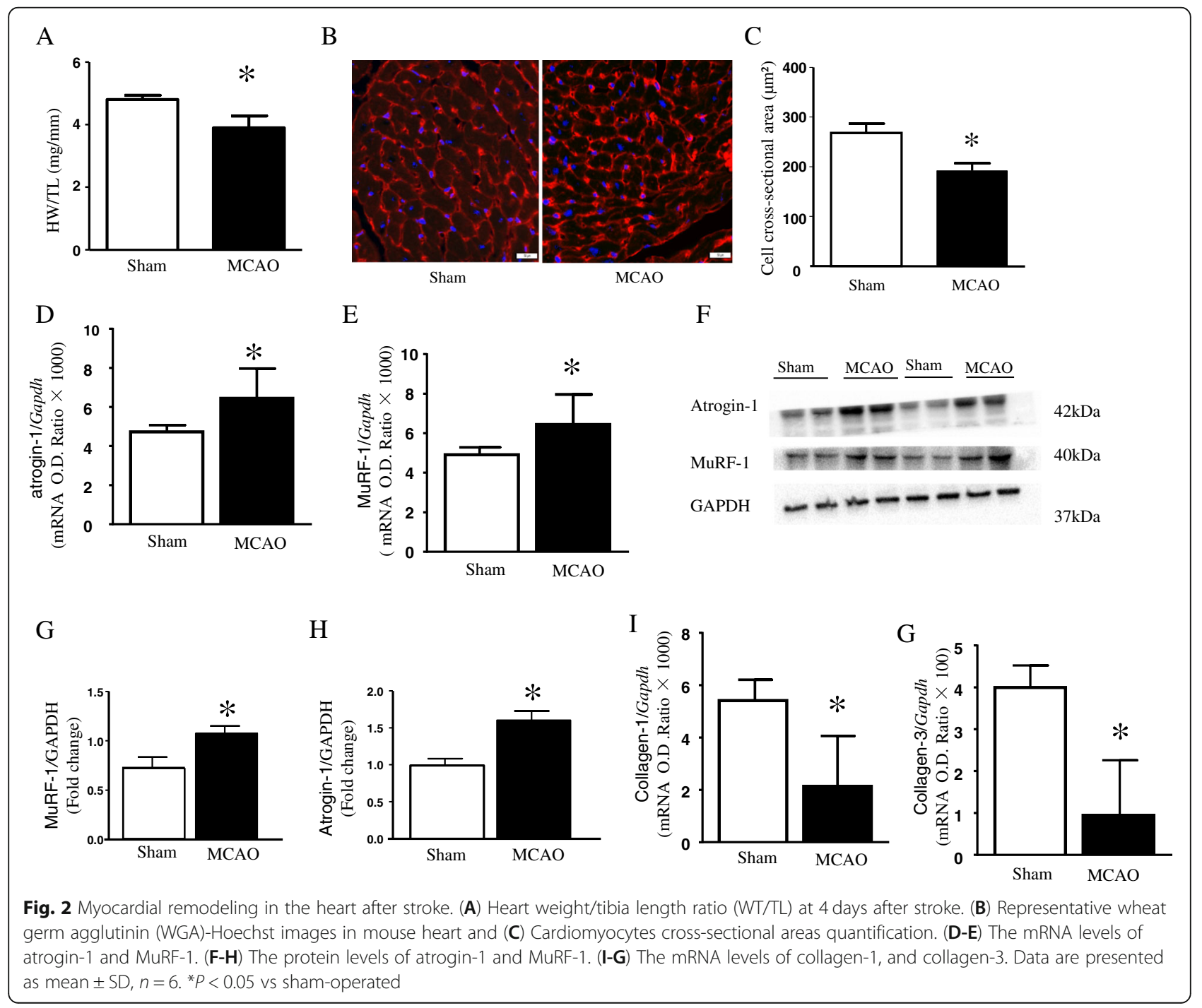


MCAO mouse hearts (Fig. 2B and C). Hematoxylin and eosin (HE) staining showed that cardiomyocytes shrank, and extracellular matrix cavities became larger in MCAO mouse tissue (Supplemental Fig. S1A). Furthermore, mRNA and protein expression levels of atrogin-1 (Fig. 2D and F) and MuRF-1 (Fig. 2F-H) were upregulated in MCAO mouse hearts (full blots see in Supplemental Fig. S3).

In addition, we detected the expression of marker genes of cardiac hypertrophy and fibrosis.. We found that hypertrophic expression of the $\beta-\mathrm{MHC}$ gene (Supplemental Fig. S1B) was upregulated, as well as the level of ANP mRNA (Supplemental Fig. S1C). Unexpectedly, the mRNA expression levels of collagen-1 (Fig. 2I) and collagen-3 (Fig. 2G), which are classically involved in pathological myocardial remodeling, were reduced in the stroke-induced mouse hearts. However, Sirius red staining did not demonstrate a change in the deposition of total collagen in the MCAO-induced mouse hearts (Supplemental Fig. S1D).

\section{DEGs in stroke-induced mouse hearts}

High throughput sequencing was performed using cardiac RNA from MCAO mouse hearts after $40 \mathrm{~h}$. First, we obtained DEGs using cutoff FC $>2$ and FC $>2$ and cutoff FC $<0.5$ for both up-/down-regulated genes between MCAO and sham-operated mice (Fig. 3A). Bioinformatics analysis revealed a total of 383 DEGs in the MCAO mouse hearts for which 221 transcripts showed decrease in abundance, while 162 genes showed an increase (Fig. 3B). We listed the top ten differentially upregulated miRNA genes and the downregulated miRNA genes in Table 1 and in Table 2 in Gene ID forms. In order to present the overall distribution of DEGs, we drawn a MA plot (Fig. 3C) and a volcano plot (Fig. 3D) with a threshold of $\log 2 \mathrm{FC}>1$. In a MA plot, significant

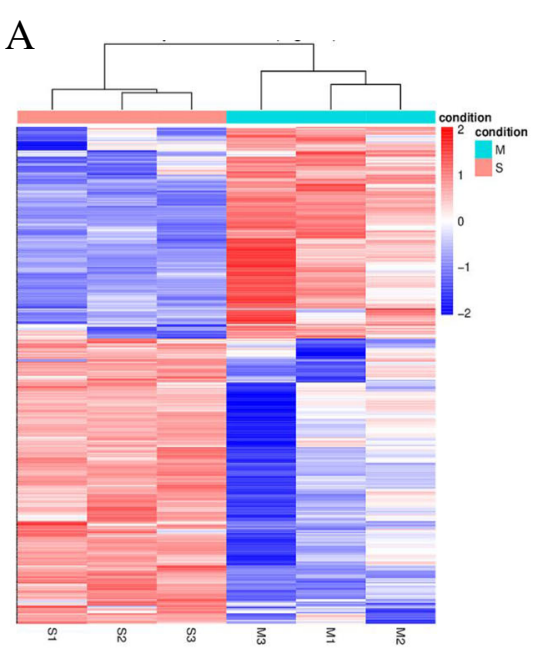

$\mathrm{C}$

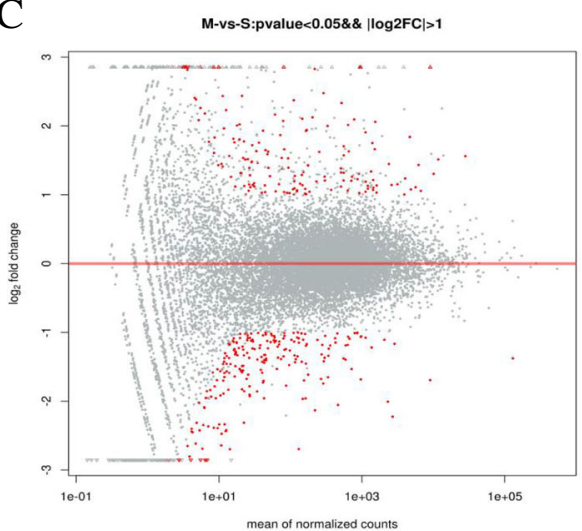

B

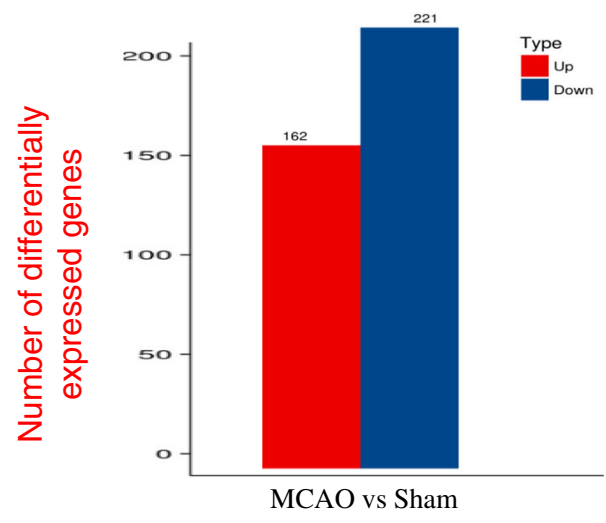

$\mathrm{D}$

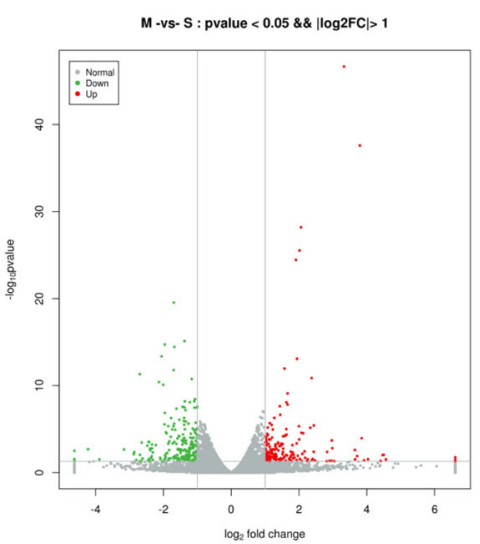

Fig. 3 Transcriptome genes expression altered in stroke-induced heart. (A) Hierarchal clustering of 383 differentially expressed genes (DEGs) in middle cerebral artery occlusion (MCAO) hearts found using RNA sequencing (RNAseq). (B) 221 DEGs were downregulated while 162 were upregulated in stroke mouse hearts. (C) DEGs separated (MA plot) in 1-fold change (FC) comparing with sham-operated group. (D) A volcano plot of DEGs 
DEGs are marked in red. In a volcano plot, significant DEGs were marked in red and green.

\section{GO analysis of DEGs in stroke-induced mouse hearts}

To further understand the gene changes associated with stroke-induced cardiomyopathy, GO enrichment analysis was performed by clusterProfiler on 2770 GO-terms, with 1405 Down-terms $(P<0.05)$ and 1063 Up-terms $(P<0.05)$.

The top 30 GO-terms of down-regulated DEGs with the highest enrichment factor are shown in Fig. 4A. Enrichment analysis showed that a total of 319 downregulated DEGs were significantly enriched in the top 30 GO-terms, 45 of which were in biological processes (BP), 237 of which were in cellular components (CC), and 37 of which were in molecular functions (MF) with $P<0.05$. The top 5 enriched BP terms were immune response, collagen fibril organization, positive regulation of interferon-gamma production, antigen processing and presentation of exogenous peptide antigen via major histocompatibility complex (MHC) class II, and antigen processing and presentation of peptide or polysaccharide antigen via MHC class II. The top 7 enriched CC terms were membrane, extracellular (region, space, and matrix), proteinaceous extracellular matrix, external side of plasma membrane, and collagen trimer. The top 5 enriched MF terms were heparin binding, collagen binding, flavin adenine dinucleotide binding, voltage-gated potassium channel activity, and integrin binding. These results showed that MCAO suppressed immune response and collagen synthesis, and the DEGs existed in the membrane or extracellular region of cardiomyocytes.

The top 30 GO-terms of up-regulated DEGs with the highest enrichment factor are shown in Fig. 4B. Enrichment analysis showed that a total of 235 up-regulated DEGs were significantly enriched in the top 30 GOterms, 39 of which were BP, 146 of which were CC, and 50 of which were MF with $P<0.05$. The top 5 enriched BP terms were negative regulation of peptidase activity, negative regulation of endopeptidase activity, steroid metabolic process, acute-phase response and response to cytokine. The top 5 enriched CC terms were cytoplasm, extracellular region, extracellular space, apical plasma membrane and secretory granule. The top 5 enriched MF terms were oxidoreductase activity, catalytic activity, peptidase inhibitor activity, flavin adenine dinucleotide binding and lyase activity. These results show that modified activity of oxidoreductase, peptidase, and endopeptidase may be involved in MCAO cardiomyopathy. The up-regulation of DEGs mainly existed in the cytoplasm and extracellular region of cardiomyocytes.

\section{KEGG analysis of DEGs in stroke mouse heart}

Pathway analysis using KEGG can provide further insights into gene functions and their interactions. The top 20 enriched pathways are presented in Fig. 5A and

Table 1 Basic and obstetric characteristics of SSI and control groups

\begin{tabular}{|c|c|c|c|c|c|}
\hline Gene Name & FC & P.Value & Length & Gene ID & Pathway \\
\hline amyloid $\mathrm{A} 3$ & 97.563782 & 0.047873262 & 122 & 20210 & \\
\hline \multirow[t]{2}{*}{ alpha-inducible protein 27 like $2 B$} & 23.636041 & 0.03066509 & & 217845 & \\
\hline & & & 283 & & \\
\hline acrosome associated 3 & 22.603644 & 0.009068478 & 163 & 75622 & \\
\hline predicted gene, 40755 & 22.087384 & 0.009506838 & 1750 & 105245278 & \\
\hline zinc finger protein 473 & 21.231115 & 0.045417807 & 892 & 243963 & \\
\hline $\begin{array}{l}\text { cytochrome P450, family } 4 \text {, } \\
\text { subfamily f, polypeptide } 15\end{array}$ & 16.400493 & 0.029365916 & 197 & 106648 & \\
\hline $\begin{array}{l}\text { Protein-L-isoaspartate } \\
\text { O-methyltransferase }\end{array}$ & 15.139410 & 0.039437647 & 363 & 100039848 & $\begin{array}{l}\text { mmu04064, } \\
\text { mmu04060, } \\
\text { mmu04062 }\end{array}$ \\
\hline $\begin{array}{l}\text { solute carrier family } 22 \\
\text { (organic anion/cation transporter) }\end{array}$ & 14.395350 & 0.000110391 & 192 & 1220521 & \\
\hline chromogranin B & 13.122352 & 0.012467959 & 677 & 12653 & \\
\hline RIKEN cDNA $2610028 \mathrm{H} 24$ gene & 12.568344 & 0.02968735 & 2594 & 76964 & \\
\hline
\end{tabular}


Table 2 Multivariate analysis of risk factors for SSI

\begin{tabular}{|c|c|c|c|c|c|}
\hline Gene Name & $\mathrm{FC}$ & P. Value & Length & Gene ID & Pathway \\
\hline integrin alpha 11 & 0.499612 & 0.03570586 & 1188 & 319480 & $\begin{array}{l}\text { mmu04151, mmu04512, } \\
\text { mmu04510, mmu04810, } \\
\text { mmu05410, mmu05412, } \\
\text { mmu05414, mmu05165 }\end{array}$ \\
\hline transmembrane protein $150 \mathrm{C}$ & 0.499301 & 0.000057427 & 249 & 231503 & \\
\hline cyclin J-like & 0.498645 & 0.033749661 & 387 & 380694 & \\
\hline $\begin{array}{c}\text { phosphatidylcholine transfer } \\
\text { protein }\end{array}$ & 0.497695 & 0.000100616 & 214 & 18559 & \\
\hline bone morphogenetic protein 7 & 0.497218 & 0.009549235 & 430 & 12162 & $\begin{array}{l}\text { mmu04350, mmu04390, } \\
\text { mmu04060, mmu04360 }\end{array}$ \\
\hline integrator complex subunit 2 & 0.497074 & 0.000394172 & 1198 & 70422 & \\
\hline $\begin{array}{c}\text { Protein associated with UVRAG } \\
\text { as autophagy enhancer }\end{array}$ & 0.495589 & 0.02455509 & 648 & 271221 & \\
\hline mesenchyme homeobox 1 & 0.494969 & 0.000040091 & 253 & 17285 & \\
\hline $\begin{array}{l}\mathrm{Cbp} / \mathrm{p} 300 \text {-interacting } \\
\text { transactivator, with Glu/Asp-rich } \\
\text { carboxy-terminal domain, } 4\end{array}$ & 0.494740 & 0.000961498 & 182 & 56222 & \\
\hline histone cluster $1, \mathrm{H} 2$ be & 0.494220 & 0.018244307 & 126 & 319179 & $\begin{array}{c}\text { mmu05203, mmu05322, } \\
\text { mmu05034 }\end{array}$ \\
\hline
\end{tabular}

FC fold change

arranged according to the number of down-regulated DEGS. The top 10 pathways of down-regulated DEGs with the greatest enrichment were cytokine-cytokine receptor interaction, protein digestion and absorption, extracellular matrix (ECM)-receptor interaction, hematopoietic cell lineage, influenza A, axon guidance, focal adhesion, inflammatory bowel disease (IBD), rheumatoid arthritis, and advanced glycation endproducts/receptor for advanced glycation end-products (AGE-RAGE) signaling pathway in diabetic complications.

The top 20 enriched pathways are presented in Fig. 5B and arranged according to the number of up-regulated DEGS. The top 10 pathways of up-regulated DEGs with the greatest enrichment were microRNAs in cancer, chemical carcinogenesis, cytokine-cytokine receptor interaction, metabolism of xenobiotics by cytochrome P450, drug metabolism-cytochrome P450, PPAR signaling pathway, autophagy-animal, fluid shear stress and atherosclerosis, hepatocellular carcinoma, herpes simplex infection and tyrosine metabolism.
Overall, the analysis of top-ranked pathways showed that genes in the collagen metabolic pathway such as Col1a1, Col4a6, and Col6a3 were suppressed, whereas those in the cytochrome P450 and PPAR signaling pathways were activated, and the cytokine-cytokine receptor interaction pathway was dysregulated in the MCAO mouse heart.

\section{Verification of DEGs by qPCR}

Based on the FC, GO, and pathway results, we selected 7 genes among all identified DEGs for verification by qPCR. As shown in Fig. 6A-D, Arrdc2, Hsd11b1, $H m g c s 2$, and $S c d 4$ were significantly up-regulated, whereas Egrl, Apln, and Aplnr were significantly downregulated in the MCAO mouse heart (Fig. 6E-G).

\section{Detection of mRNA of proinflammatory factor}

In order to further observe the similarity of inflammatory response in heart and brain, we detected the mRNA levels of IL1 $\beta$, IL-6, TNF- $\alpha$. Whether in the heart or the 


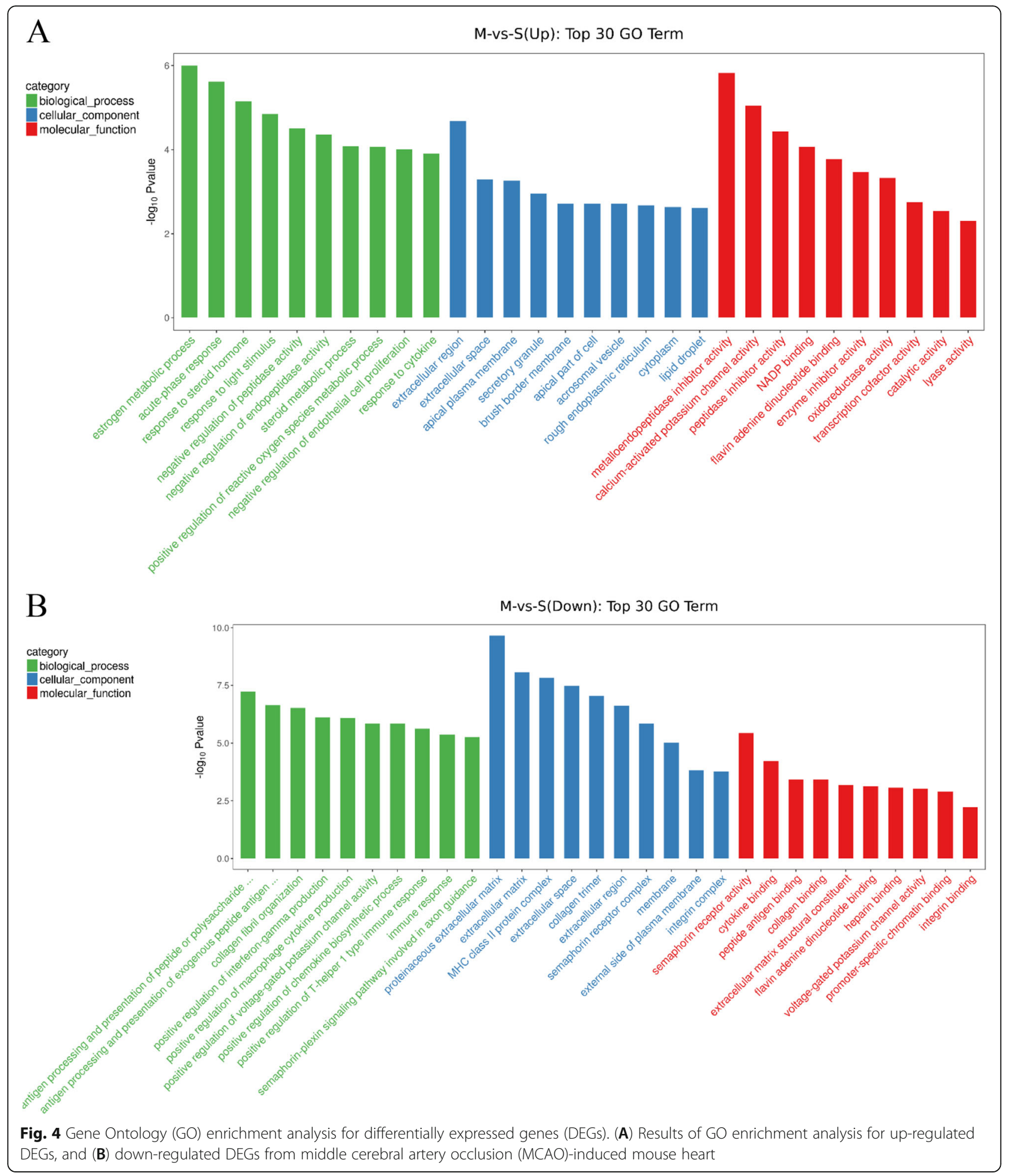


A

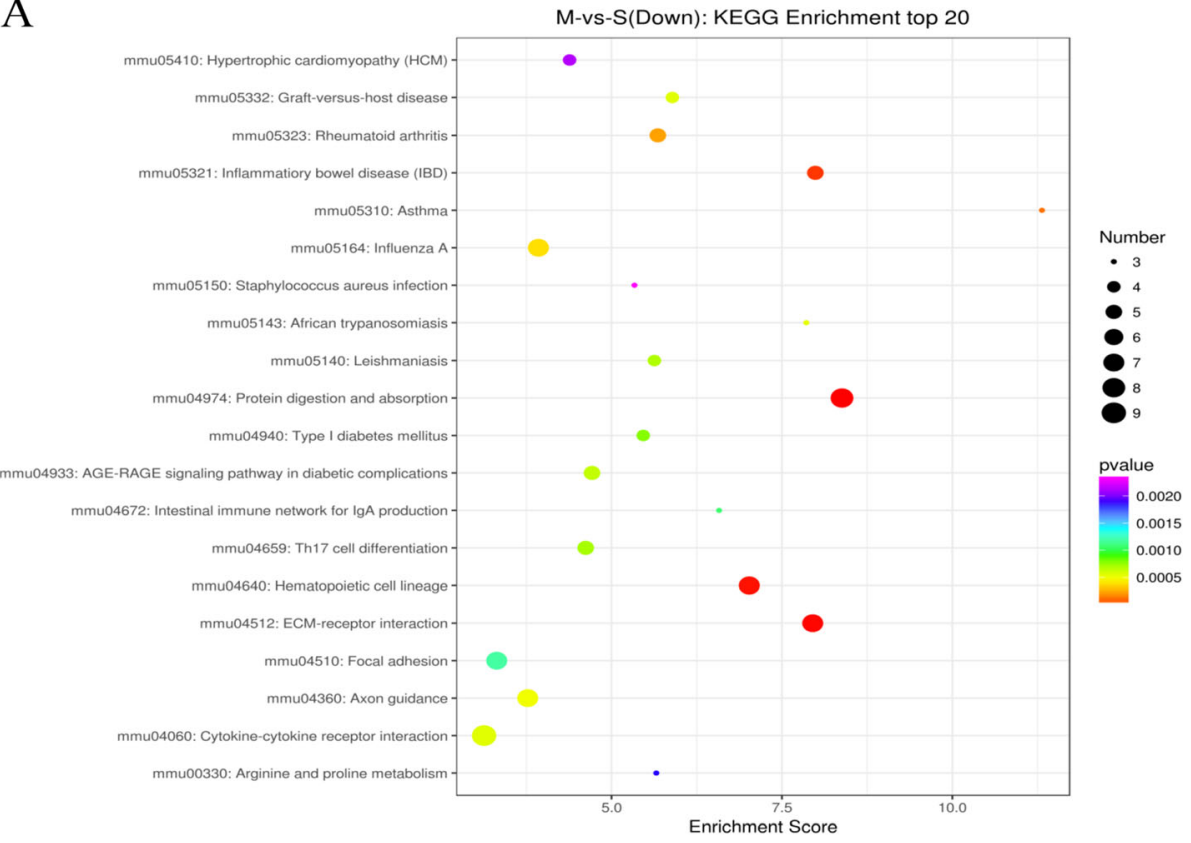

$\mathrm{B}$

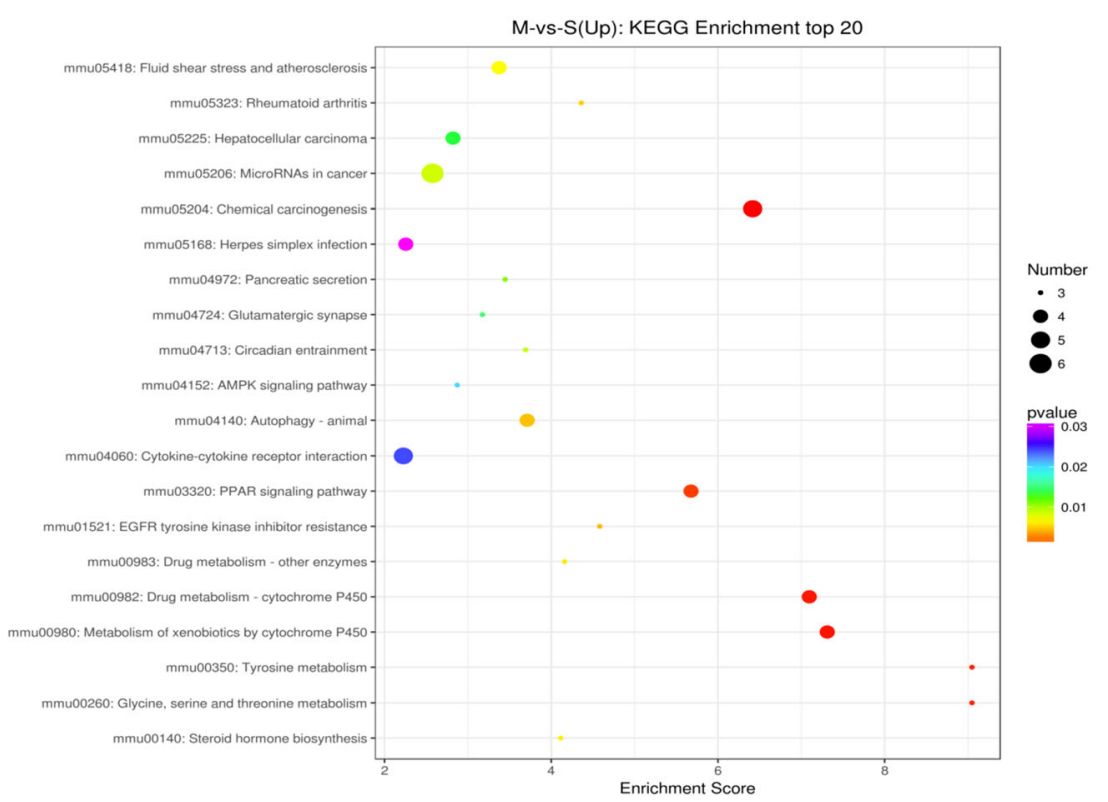

Fig. 5 Kyoto Encyclopedia of Genes and Genomes (KEGG) enrichment analysis for differentially expressed genes (DEGs). (A) Results of KEGG enrichment analysis for down-regulated DEGs, and (B) up-regulated DEGs in middle cerebral artery occlusion (MCAO)-induced mouse heart. Bubble chart of the top 20 significantly enriched pathways for the down-regulated DEGs

brain, the expression of IL1 $\beta$, IL-6, TNF- $\alpha$ mRNA was up-regulated in MCAO mouse hearts (Fig. 7).

\section{Discussion}

Despite the existence of cardioembolic stroke [26], there is sufficient evidence in many clinical and animal investigations indicating that stroke could induce cardiac injury
[5, 10, 27]. However, little is known about the mechanism of stroke-induced cardiomyopathy. This study revealed three major new findings: (1) experimental induced stroke caused cardiac dysfunction at $4 \mathrm{~d}$; (2) atrophic phenotype of the heart occurred after induced stroke; and (3) a unique myocardial mRNA expression profile was produced after induced stroke. 

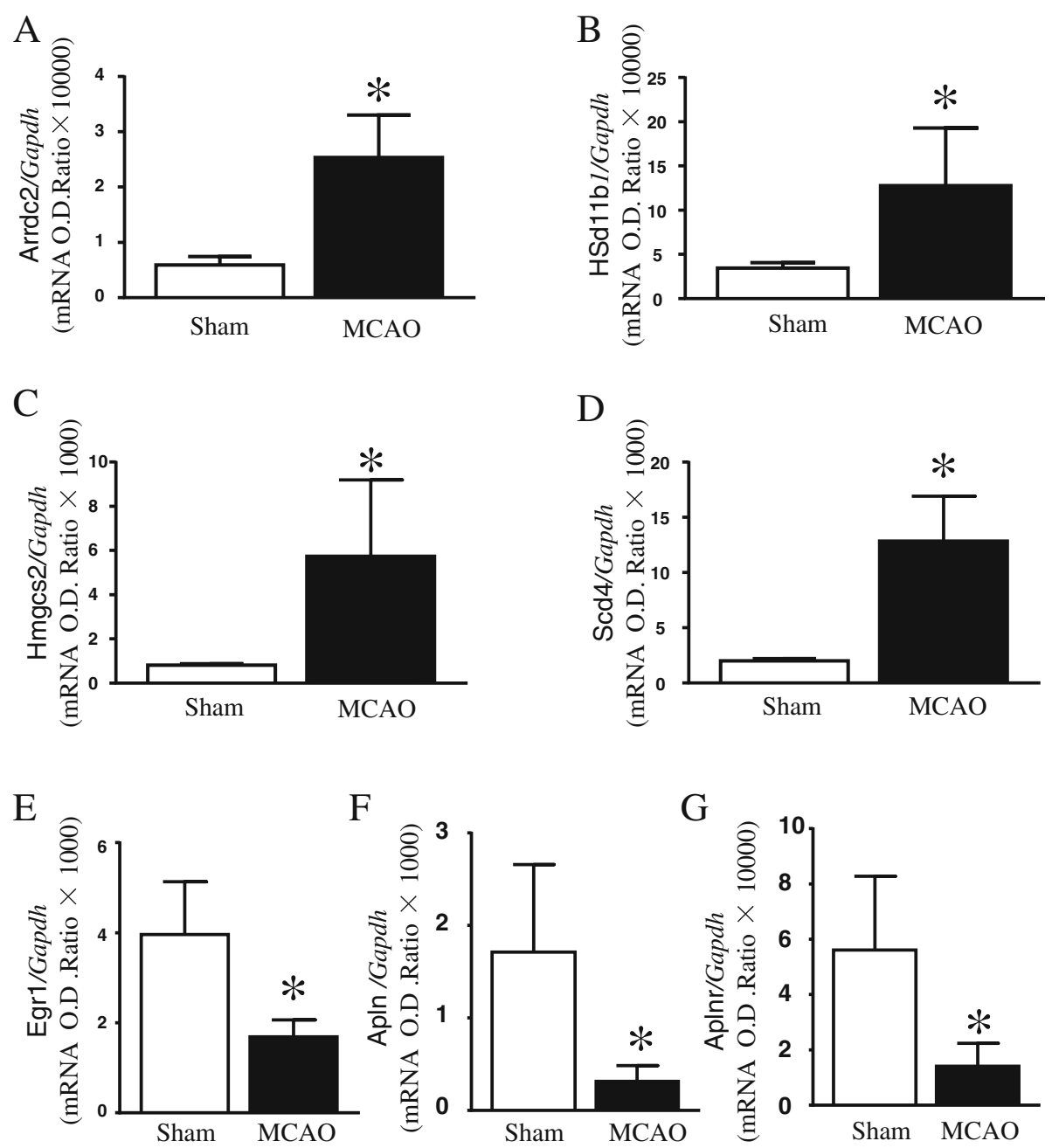

Fig. 6 Quantitative assessment of identified differentially expressed genes (DEGs) in mouse heart. Expression of the identified DEGs from RNAseq experiments were quantified using quantitative PCR (qPCR). (A-D) mRNA expression of Arrdc2, Hsd1 1b1, Hmgcs2, and Scd4 in mouse heart. (E-G) mRNA expression of Egr1, Apln, and Aplnr in mouse heart. Data are presented as mean $\pm \mathrm{SD}, n=6 .{ }^{*} P<0.05$ vs sham-operated

Stroke induced progressive cardiac dysfunction in adult mice

Cardiac dysfunction is frequently encountered in various forms of stroke such as ischemic stroke [10], brain hemorrhage [28] and subarachnoid hemorrhage [29]. Therefore, neural deregulation and systemic inflammation are considered to be the common mechanism of brain-heart interaction $[11,30]$. Based on the mechanism of neuro-regulation mediated cardiac function, cardiac dysfunction occurs in the acute phase. Approximately $67 \%$ of acute ischemic stroke patients have ischemic and/or arrhythmic electrocardiogram abnormalities in the first $24 \mathrm{~h}$ after a stroke [31]. During the first 3 months after acute ischemic stroke, $19.0 \%$ of patients suffer from at least one serious cardiac adverse event; $28.5 \%$ have LVEF of less than 50\%; and 13-29\% have systolic dysfunction [32]. In animal experiments, 28 days after stroke, significantly decreased cardiac function was identified by decreased LVEF compared to non-stroke mice [7]. However, other studies suggest that left ventricular contractility was reduced earlier (24-72 h) but not later ( 2 months) after brain ischemia in mice subjected to filament-induced left MCAO [12].

Impaired cardiac function as a consequence of severe acute ischemic stroke is a predictor of worse functional outcome and also contributes substantially to the longterm prognosis of ischemic stroke patients [33]. Therefore, investigating the early stage of brain-heart interaction after stroke is highly clinically significant. From other longitudinal studies, cardiac function was maximally decreased within 2-4 days after MCAO [12]. And although we selected the appropriate monofilament, the location and extent of neurological deficit and infarction in MCAO mice in the same group may not be 

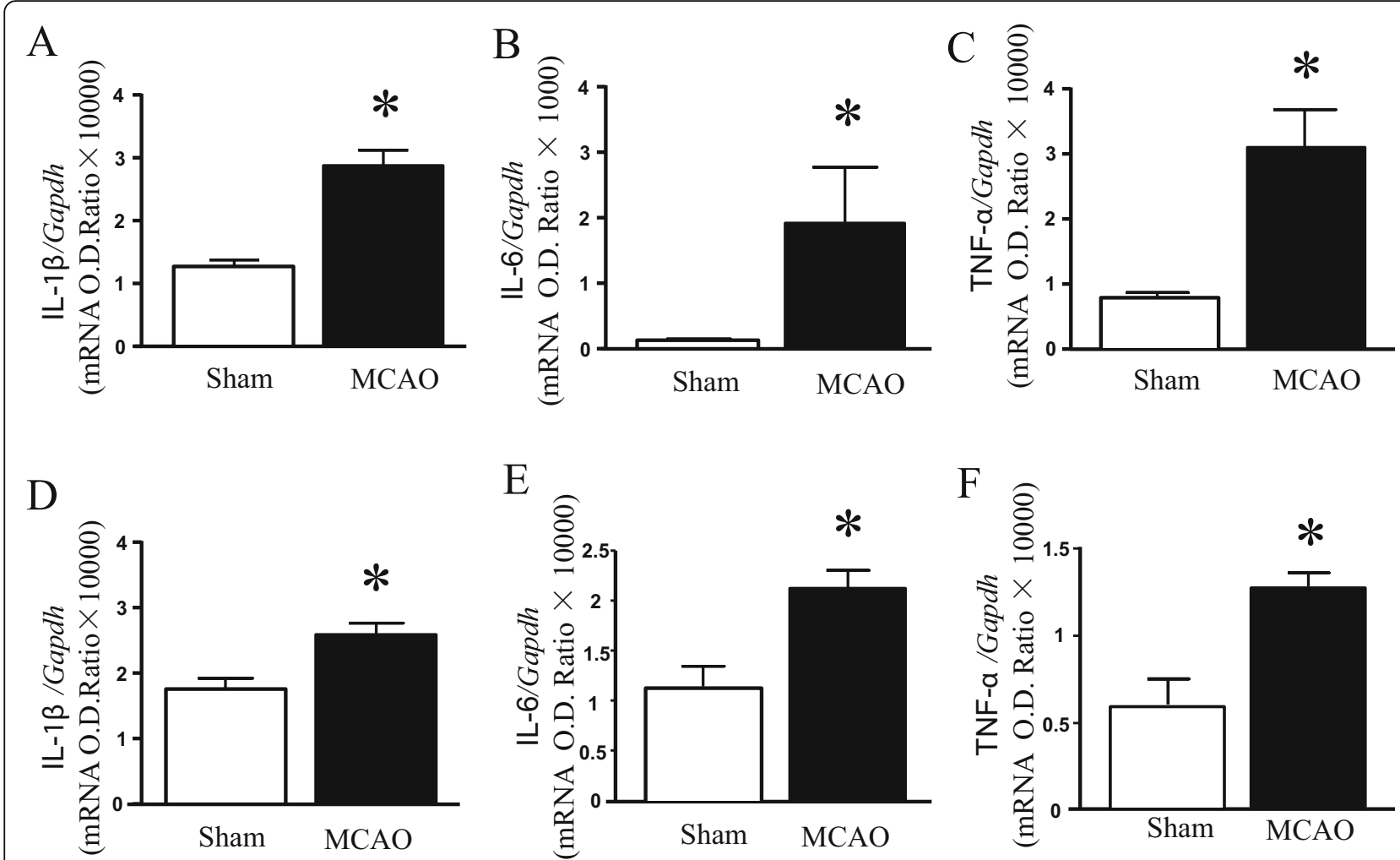

Fig. 7 Expressions of pro-inflammatory factor mRNAs between heart and brain in mouse. (A-C) The levels of IL1 $\beta$, IL-6, TNF-a mRNA in the heart. (D-F) The levels of IL1 $\beta$, IL-6, TNF-a mRNA in the brain

consistent due to the difference of vascular diameter. From the side, a certain degree of cerebral infarction will produce adverse effects on cardiac function and injury.

In our experimental study, we found a substantial reduction of $\mathrm{EF} \%, \mathrm{FS} \%$, and $\mathrm{E} / \mathrm{A}$ ratio in the early and subacute phase after stroke in previously healthy mice with intact cardiac function, with the decreased total blood flow and the decreased coronary reserve capacity. Our results suggest that close monitoring for cardiac dysfunction is necessary during the early stage. However, further study is needed to determine if cardiac therapy during the early stage can accelerate cerebral ischemia recovery.

\section{Stroke leads to cardiac atrophy and myocardial remodeling}

Cardiac dysfunction relates to the morphological phenotype change in mice after stroke. Previous studies reported cardiac hypertrophy as a long-term (8 week) cardiac consequence of a focal cerebral ischemia [9]. Furthermore, myocyte cross-sectional area was significantly increased compared to non-stroke mice 28 days after stroke. In contrast, mice in our study (MCAO, 60 min ischemia) showed that heart weight rapidly decreased and cardiomyocyte size reduced at 4 days. This was consistent with other researcher who also detected a reduced heart weight/tibia length ratio and cardiomyocyte cross-sectional diameter, which normalized after 14 days [12]. Cardiac phenotype observed in stroke-induced mouse heart did not result in myocardial hypertrophy, but this might have changed over time with deterioration of heart function.

Additionally, expression of collagens that are normally involved in pathological cardiac remodeling was not increased (collagen-1 and collagen-3) in our inducedstroke mouse heart. Furthermore, GO and pathway analyses indicated that stroke induced collagen synthesis related DEGs were down-regulated. A recent study which was consistent with our results, reported that the expression of col3a1 was reduced, and that of the E3 ubiquitin ligase atrogin-1 and muscle ring finger protein MuRF-1, which are known to regulate skeletal muscle and cardiac atrophy, was increased in stroke-induced mouse heart [12]. Previously, tail-suspension for 28 days, which simulated spaceflight or microgravity conditions, decreased cardiomyocyte size and heart weight, increased MuRF-1 protein levels, and cause myocardial atrophy and dysfunction [34]. Recent animal study data indicate that anthracycline-caused cardiac atrophy is dependent on MuRF-1 [35]. The changes detected at the early stage of stroke showed remodeling characteristics typical of myocardial atrophy as observed due to 
weightlessness and cancer [36]. MuRF-1 and collagen may be very important mediating mechanisms. However, whether the treatment of short-term myocardial atrophy can prevent long-term myocardial fibrosis and hypertrophy, deserves further investigation.

\section{Stroke altered transcriptome characteristics}

Acute ischemic and hemorrhagic strokes in some particular areas of the brain can disrupt central autonomic control of the heart, precipitating cardiac arrhythmias, myocardial injury and sometimes sudden death [37]. Therapeutically, beta-blockade with metoprolol inhibits sympathetic activity, which then prevents the development of chronic cardiac dysfunction by decelerating cardiac remodeling in ischemic stroke mice [9]. Further study is needed on the heart effects caused by stroke, neural regulation, and cardiomyocyte changes produced at the cell and biological levels.

Stroke induced cardiomyopathy has a unique transcriptome expression profile, different from myocardial infarction [38] and cardiac hypertrophy [39]. Veltkamp [12] reported that several DEGs were up-regulated in the heart $24 \mathrm{~h}$ after stroke. However, we found that there were more down-regulated DEGs in MCAO-induced mouse heart. The most significant feature was that stroke-induced hearts had an inhibited immune response. Further, DEGs of cytokine-cytokine receptor interaction, including Il11, Il18rap, Il22ra1, and Il6ra, were moderately increased. These findings support recent reports that splenectomy with stroke significantly reduces macrophage infiltration into the heart, decreases inflammatory factor expression in the heart, and significantly improves cardiac function compared to nonsplenectomized adult stroke mice [11]. Further, Cxcr4 deficiency reduces innate-immune-system-mediated defense response, which is associated with a deteriorating outcome after transient cerebral ischemia [40].

Unexpectedly, unlike the common pathological remodeling, many collagen metabolic genes were decreased in the stroke-induced heart. These DEGs are mainly distributed in the extracellular matrix, extracellular region, and collagen trimer. Correspondingly, negative regulation of peptidase and endopeptidase was increased in MCAO mouse heart, which may further reduce collagen content. Similarly, autophagy, a protein degradation pathway, was shown to be involved in cardiac dysfunction regulation and tail-suspension induced atrophy. Therefore, disruption of the collagen pathway may play an important role in the cardiac atrophy phenotype. Meanwhile, we found that proinflammatory factors increased in both brain and heart, which is consistent with our sequencing results. The brain-heart interactions pathways disrupted by increased proinflammatory responses and reduced immunocompet- ence may be one of the important mechanisms of stroke-induced cardiomyopathy.

In addition, DEGs for oxidoreductase and catalytic activities in the cytoplasm were increased. Of these, we verified that $H s d 11 b 1$ and $H m g c s 2$ were consistent with the sequencing results. These results suggested that activated protein degradation pathway and inhibited collagen reduction caused by stroke contribute to myocardial atrophy. Importantly, we also confirmed that the expression of Apln and Aplnr significantly decreased after stroke.

The expression of apelin was reduced in an agedependent manner in humans and rodents and was positively associated with the beneficial effects of exercise in older adults [41]. Tail-suspension for 28 days decreased cardiomyocyte size and heart weight, and reduced expression of Apln and Aplnr [34]. It was also reported that endogenous apelin plays a crucial role in suppressing angiotensin II-induced cardiac dysfunction and pathological remodeling [42]. However, apelin as the target of an innovative pharmacological strategy to prevent stroke-associated myocardial atrophy needs further research.

In conclusion, the experimental induced stroke caused cardiac dysfunction has been well proved and atrophic phenotype of the heart may be an important factor leading to stroke induced cardiomyopathy. Combined with a unique myocardial mRNA sequencing, we screened the differentially expressed miRNA genes that may lead to myocardial atrophy or myocardial dysfunction. To explore the exact relationship between stroke and cardiomyopathy and the interactions between various mediators is the focus of our next study.

\section{Supplementary Information}

The online version contains supplementary material available at https://doi. org/10.1186/s12864-021-07938-y.

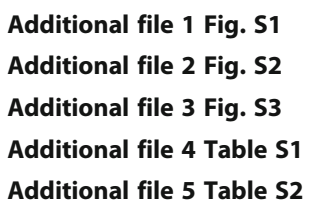

Acknowledgments

We thank Maopin Chu, The Second Affiliated Hospital and Yuying Children's Hospital of Wenzhou Medical University, Wenzhou, Zhejiang, China, for providing the test equipment of echocardiography.

\section{Authors' contributions}

J.C., J.H.G, and H.L.C. performed experiments; X.Q.L., W.L. and X.L.Q. analyzed the data; K.C.Z. and T.W. interpreted the results of experiments; S.C.L., L. L, and S.H.J. approved the final version of the manuscript.

\section{Funding}

This work was funded by funds of the National Natural Science Foundation of China (nos. 81802243, 81873376), Basic Research Program of Wenzhou 
City (Y20190079), and the Yuying Research Program of the Second Affiliated Hospital and Yuying Children's Hospital of Wenzhou Medical University.

\section{Availability of data and materials}

All supporting data can be found within the manuscript and its supplementary files. The datasets analysed during the current study are available in the NCBI under the accession number PRJNA731991 [https:// www.ncbi.nlm.nih.gov/sra/PRJNA731991]. And the corresponding author Dr. Li can be contacted to access the data by email (lishengcun@wmu.edu.cn).

\section{Declarations}

\section{Ethics approval and consent to participate}

All experiments were approved by the Institutional Animal Care and Use Committee of Wenzhou Medical University. We performed these experiments under the ARRIVE (Animal Research: Reporting of In Vivo Experiments) guidelines

\section{Consent for publication}

Not applicable.

\section{Competing interests}

The authors declare that they have no competing interests.

\section{Author details}

${ }^{1}$ Rehabilitation Medicine Center, The Second Affiliated Hospital and Yuying Children's Hospital of Wenzhou Medical University, Wenzhou 325027 Zhejiang, China. ${ }^{2}$ Integrative \& Optimized Medicine Research center, China-USA Institute for Acupuncture and Rehabilitation, Wenzhou Medical University, Wenzhou 325027, Zhejiang, China. ${ }^{3}$ Key Laboratory of Chinese Internal Medicine of Ministry of Education and Beijing, Dongzhimen Hospital, Beijing University of Chinese Medicine, Beijing, China. ${ }^{4}$ Institute of Cardiovascular Development and Translational Medicine, The Second Affiliated Hospital and Yuying Children's Hospital of Wenzhou Medical University, Wenzhou, Zhejiang, China.

\section{Received: 27 February 2021 Accepted: 14 August 2021}

\section{Published online: 04 September 2021}

References

1. GBDS. C: global, regional, and national burden of stroke, 1990-2016: a systematic analysis for the global burden of disease study 2016. Lancet Neurol. 2019;18(5):439-58.

2. Chen Z, Venkat P, Seyfried D, Chopp M, Yan T, Chen J. Brain-heart interaction: cardiac complications after stroke. Circ Res. 2017;121(4):451-68. https://doi.org/10.1161/CIRCRESAHA.117.311170.

3. Otaki Y, Watanabe T, Sato N, Shirata T, Tsuchiya H, Wanezaki M, et al. Direct comparison of prognostic ability of cardiac biomarkers for cardiogenic stroke and clinical outcome in patients with stroke. Heart Vessel. 2019;34(7): 1178-86. https://doi.org/10.1007/s00380-019-01345-w.

4. Yaghi S, Chang AD, Ricci BA, Jayaraman MV, McTaggart RA, Hemendinger $M$, et al. Early elevated troponin levels after ischemic stroke suggests a Cardioembolic source. Stroke. 2018;49(1):121-6. https://doi.org/10.1161/ STROKEAHA.117.019395.

5. Jordan K, Yaghi S, Poppas A, Chang AD, Mac Grory B, Cutting S, et al. Left atrial volume index is associated with Cardioembolic stroke and atrial fibrillation detection after embolic stroke of undetermined source. Stroke. 2019;50(8):1997-2001. https://doi.org/10.1161/STROKEAHA.119.025384.

6. Chang A, Ricci B, Grory BM, Cutting S, Burton T, Dakay K, et al. Cardiac biomarkers predict large vessel occlusion in patients with ischemic stroke. J Stroke Cerebrovasc Dis. 2019;28(6):1726-31. https://doi.org/10.1016/j. jstrokecerebrovasdis.2019.02.013.

7. Chen J, Cui C, Yang X, Xu J, Venkat P, Zacharek A, et al. MiR-126 affects brain-heart interaction after cerebral ischemic stroke. Transl Stroke Res. 2017 8(4):374-85. https://doi.org/10.1007/s12975-017-0520-z.

8. Li W, Li L, Chopp M, Venkat P, Zacharek A, Chen Z, et al. Intracerebral hemorrhage induces cardiac dysfunction in mice without primary cardiac disease. Front Neurol. 2018:9:965. https://doi.org/10.3389/fneur.2018.00965.

9. Bieber M, Werner RA, Tanai E, Hofmann U, Higuchi T, Schuh K, et al. Strokeinduced chronic systolic dysfunction driven by sympathetic overactivity. Ann Neurol. 2017:82(5):729-43. https://doi.org/10.1002/ana.25073.
10. Meloux A, Rigal E, Rochette L, Cottin $Y$, Bejot $Y$, Vergely C. Ischemic stroke increases heart vulnerability to ischemia-reperfusion and alters myocardia Cardioprotective pathways. Stroke. 2018;49(11):2752-60. https://doi.org/1 $0.1161 /$ STROKEAHA.118.022207.

11. Yan T, Chen Z, Chopp M, Venkat P, Zacharek A, Li W, et al. Inflammatory responses mediate brain-heart interaction after ischemic stroke in adult mice. J Cereb Blood Flow Metab. 2020;40(6):1213-29. https://doi.org/10.11 77/0271678X18813317.

12. Veltkamp R, Uhlmann S, Marinescu M, Sticht C, Finke D, Gretz N, et al. Experimental ischaemic stroke induces transient cardiac atrophy and dysfunction. J Cachexia Sarcopenia Muscle. 2019;10(1):54-62. https://doi. org/10.1002/jcsm.12335

13. Liu H, Xie Q, Xin BM, Liu JL, Liu Y, Li YZ, et al. Inhibition of autophagy recovers cardiac dysfunction and atrophy in response to tail-suspension. Life Sci. 2015:121:1-9. https://doi.org/10.1016/j.lfs.2014.10.023.

14. Saand AR, Yu F, Chen J, Chou SH. Systemic inflammation in hemorrhagic strokes - a novel neurological sign and therapeutic target? J Cereb Blood Flow Metab. 2019;39(6):959-88. https://doi.org/10.1177/0271678X19841443.

15. Zhou K, Chen J, Wu J, Wu Q, Jia C, Xu YXZ, et al. Atractylenolide III ameliorates cerebral ischemic injury and neuroinflammation associated with inhibiting JAK2/STAT3/Drp1-dependent mitochondrial fission in microglia. Phytomedicine. 2019;59:152922. https://doi.org/10.1016/j.phymed.2019.152 922.

16. Cao T, Fan S, Zheng D, Wang G, Yu Y, Chen R, et al. Increased calpain-1 in mitochondria induces dilated heart failure in mice: role of mitochondrial superoxide anion. Basic Res Cardiol. 2019;114(3):17. https://doi.org/10.1007/ s00395-019-0726-1.

17. Teng X, Ji C, Zhong H, Zheng D, Ni R, Hill DJ, et al. Selective deletion of endothelial cell calpain in mice reduces diabetic cardiomyopathy by improving angiogenesis. Diabetologia. 2019;62(5):860-72. https://doi.org/1 0.1007/s00125-019-4828-y.

18. Li S, Zhang L, Ni R, Cao T, Zheng D, Xiong S, et al. Disruption of calpain reduces lipotoxicity-induced cardiac injury by preventing endoplasmic reticulum stress. Biochim Biophys Acta. 2016;1862(11):2023-33. https://doi. org/10.1016/j.bbadis.2016.08.005.

19. Longa EZ, Weinstein PR, Carlson S, Cummins R. Reversible middle cerebral artery occlusion without craniectomy in rats. Stroke. 1989;20(1):84-91. https://doi.org/10.1161/01.STR.20.1.84.

20. Song $N$, Ma L, Wang W, Sun H, Wang L, Baldwin IT, et al. An ERF2-like transcription factor regulates production of the defense sesquiterpene capsidiol upon Alternaria alternata infection. J Exp Bot. 2019;70(20):5895908. https://doi.org/10.1093/jxb/erz327.

21. Roberts A, Trapnell C, Donaghey J, Rinn JL, Pachter L. Improving RNA-Seq expression estimates by correcting for fragment bias. Genome Biol. 2011; 12(3):R22. https://doi.org/10.1186/gb-2011-12-3-r22.

22. Anders S, Pyl PT, Huber W. HTSeq--a Python framework to work with highthroughput sequencing data. Bioinformatics. 2015:31(2):166-9. https://doi. org/10.1093/bioinformatics/btu638

23. Galili T. Dendextend: an R package for visualizing, adjusting and comparing trees of hierarchical clustering. Bioinformatics. 2015;31(22):3718-20. https:// doi.org/10.1093/bioinformatics/btv428.

24. Kanehisa M, Goto S. KEGG: Kyoto encyclopedia of genes and genomes. Nucleic Acids Res. 2000;28(1):27-30. https://doi.org/10.1093/nar/28.1.27.

25. Tabish AM, Arif M, Song T, Elbeck Z, Becker RC, Knöll R, et al. Association of intronic DNA methylation and hydroxymethylation alterations in the epigenetic etiology of dilated cardiomyopathy. Am J Physiol Heart Circ Physiol. 2019;317(1):H168-h180. https://doi.org/10.1152/ajpheart.00758.2018.

26. Nelson SE, Ament Z, Wolcott Z, Gerszten RE, Kimberly WT. Succinate links atrial dysfunction and cardioembolic stroke. Neurology. 2019;92(8):e802-10. https://doi.org/10.1212/WNL.0000000000006957.

27. Prasad Hrishi A, Ruby Lionel K, Prathapadas U. Head rules over the heart: cardiac manifestations of cerebral disorders. Indian J Crit Care Med. 2019: 23(7):329-35. https://doi.org/10.5005/jp-journals-10071-23208.

28. Elder T, Raghavan A, Smith A, Wright $\mathrm{CH}$, Wright J, Burant $\mathrm{C}$, et al. Outcomes after intracranial hemorrhage in patients with left ventricular assist devices: a systematic review of literature. World Neurosurg. 2019;132: 265-72. https://doi.org/10.1016/j.wneu.2019.08.211.

29. Zhang L, Wang Z, Qi S. Cardiac troponin elevation and outcome after subarachnoid hemorrhage: a systematic review and Meta-analysis. J Stroke Cerebrovasc Dis. 2015:24(10):2375-84. https://doi.org/10.1016/j. jstrokecerebrovasdis.2015.06.030. 
30. Ay H, Koroshetz WJ, Benner T, Vangel MG, Melinosky C, Arsava EM, et al. Neuroanatomic correlates of stroke-related myocardial injury. Neurology. 2006;66(9):1325-9. https://doi.org/10.1212/01.wnl.0000206077.13705.6d.

31. Purushothaman S, Salmani D, Prarthana KG, Bandelkar SM, Varghese S.

Study of ECG changes and its relation to mortality in cases of cerebrovascular accidents. J Nat Sci Biol Med. 2014;5(2):434-6. https://doi. org/10.4103/0976-9668.136225.

32. Prosser J, MacGregor L, Lees KR, Diener HC, Hacke W, Davis S. Predictors of early cardiac morbidity and mortality after ischemic stroke. Stroke. 2007; 38(8):2295-302. https://doi.org/10.1161/STROKEAHA.106.471813.

33. Gattringer T, Niederkorn K, Seyfang L, Seifert-Held T, Simmet N, Ferrari J, et al. Myocardial infarction as a complication in acute stroke: results from the austrian stroke unit registry. Cerebrovasc Dis. 2014;37(2):147-52. https:// doi.org/10.1159/000357799.

34. Liang L, Yuan W, Qu L, Li H, Zhang L, Fan GC, et al. Administration of losartan preserves cardiomyocyte size and prevents myocardial dysfunction in tail-suspended mice by inhibiting p47(phox) phosphorylation, NADPH oxidase activation and MuRF1 expression. J Transl Med. 2019;17(1):279. https://doi.org/10.1186/s12967-019-2021-1.

35. Willis MS, Parry TL, Brown DI, Mota Rl, Huang W, Beak JY, et al. Doxorubicin exposure causes subacute cardiac atrophy dependent on the striated muscle-specific ubiquitin ligase MuRF1. Circ Heart Fail. 2019;12(3):e005234. https://doi.org/10.1161/CIRCHEARTFAILURE.118.005234.

36. Murphy KT. The pathogenesis and treatment of cardiac atrophy in cancer cachexia. Am J Physiol Heart Circ Physiol. 2016;310(4):H466-77. https://doi. org/10.1152/ajpheart.00720.2015

37. Ruthirago D, Julayanont P, Tantrachoti P, Kim J, Nugent K. Cardiac arrhythmias and abnormal electrocardiograms after acute stroke. Am J Med Sci. 2016;351(1):112-8. https://doi.org/10.1016/j.amjms.2015.10.020.

38. Wang C, Bai X, Liu S, Wang J, Su Z, Zhang W, et al. RNA-seq based transcriptome analysis of the protective effect of compound longmaining decoction on acute myocardial infarction. J Pharm Biomed Anal. 2018;158: 339-45. https://doi.org/10.1016/j.jpba.2018.06.016.

39. Newman MS, Nguyen T, Watson MJ, Hull RW, Yu HG. Transcriptome profiling reveals novel BMI- and sex-specific gene expression signatures for human cardiac hypertrophy. Physiol Genomics. 2017;49(7):355-67. https:// doi.org/10.1152/physiolgenomics.00122.2016.

40. Werner Y, Mass E, Ashok Kumar P, Ulas T, Händler K, Horne A, et al. Cxcr4 distinguishes HSC-derived monocytes from microglia and reveals monocyte immune responses to experimental stroke. Nat Neurosci. 2020;23(3):351-62. https://doi.org/10.1038/s41593-020-0585-y.

41. Vinel C, Lukjanenko L, Batut A, Deleruyelle S, Pradère JP, Le Gonidec S, et al. The exerkine apelin reverses age-associated sarcopenia. Nat Med. 2018;24(9): 1360-71. https://doi.org/10.1038/s41591-018-0131-6.

42. Sato T, Kadowaki A, Suzuki T, Ito H, Watanabe H, Imai Y, Kuba K. Loss of Apelin Augments Angiotensin II-Induced Cardiac Dysfunction and Pathological Remodeling. Int J Mol Sci .2019;20(2):239. https://doi.org/10.33 90/ijms20020239.

\section{Publisher's Note}

Springer Nature remains neutral with regard to jurisdictional claims in published maps and institutional affiliations.

Ready to submit your research? Choose BMC and benefit from:
- fast, convenient online submission
- thorough peer review by experienced researchers in your field
- rapid publication on acceptance
- support for research data, including large and complex data types
- gold Open Access which fosters wider collaboration and increased citations
- maximum visibility for your research: over 100M website views per year
At BMC, research is always in progress.
Learn more biomedcentral.com/submissions

